Original Article

\title{
The Usefulness of Positron-Emission Tomography Findings in the Management of Anterior Mediastinal Tumors
}

\author{
Akihiko Kitami, MD, ${ }^{1}$ Fumitoshi Sano, MD, ${ }^{1}$ Shinichi Ohashi, MD, ${ }^{1}$ Kosuke Suzuki, MD, ${ }^{1}$ \\ Shugo Uematsu, MD, ${ }^{1}$ Takashi Suzuki, MD, ${ }^{2}$ and Mitsutaka Kadokura, MD $^{1,2}$
}

\begin{abstract}
Purpose: We performed a retrospective analysis to evaluate the usefulness of positronemission tomography/computed tomography (PET/CT) findings in the classification and management of anterior mediastinal tumors.

Methods: Between 2006 and 2015, 105 patients with anterior mediastinal tumor received PET/CT. ${ }^{18}$ F-fluorodeoxyglucose $\left({ }^{18} \mathrm{~F}\right.$-FDG)-PET images were obtained 60 minutes after the injection of ${ }^{18}$ F-FDG.

Results: The histological classifications were as follows: thymoma $(n=49)$, thymic carcinoma (TC) $(n=19)$, malignant lymphoma $(M L)(n=8)$, teratoma $(n=7)$, thymic cyst $(n=14)$, and others $(n=8)$. Upon visual inspection (SUV max: $>2.0$ ), all of the malignant tumors showed ${ }^{18}$ F-FDG accumulation (with the exception of one type $A$ thymoma). Two of the 14 thymic cysts and three of the seven teratomas showed slight ${ }^{18}$ F-FDG accumulation. The SUV max values of the low-grade thymomas, high-grade thymomas, TCs and MLs were 3.14 \pm 0.73 , $4.34 \pm 1.49,8.59 \pm 3.05$, and $10.08 \pm 2.53$, respectively, with significant differences between the low- and high-grade thymomas, and between TCs and MLs. The sensitivity, specificity and accuracy of ${ }^{18}$ F-FDG in the detection of low-grade thymomas and thymomas with a maximum diameter of $\leq 50 \mathrm{~mm}$ and an SUV max of $\leq 3.4$ were $85 \%, 48 \%$, and $60 \%$, respectively. Conclusion: FDG-PET/CT is an objective and useful modality in the differential diagnosis and management of anterior mediastinal tumors.
\end{abstract}

Keywords: FDG-PET/CT, SUV max, anterior mediastinal tumors, thymoma

\section{Introduction}

The utility of fluorodeoxyglucose-positron-emission tomography (FDG-PET) has been investigated in thymic

${ }^{1}$ Respiratory Disease Center, Showa University Northern Yokohama Hospital, Yokohama, Kanagawa, Japan

${ }^{2}$ Division of Chest Surgery, Showa University School of Medicine, Tokyo, Japan

Received: September 6, 2016; Accepted: November 7, 2016 Corresponding author: Akihiko Kitami, MD. Respiratory Disease Center, Showa University Northern Yokohama Hospital, 35-1 Chigasaki-Chuo, Tsuzuki-ku, Yokohama, Kanagawa 224-8503, Japan Email: akitami@med.showa-u.ac.jp

(C)2017 The Editorial Committee of Annals of Thoracic and Cardiovascular Surgery. All rights reserved. epithelial tumors. ${ }^{1-7)}$ However, little is known about the usefulness of FDG-PET imaging in anterior mediastinal lesions that are not thymomas or thymic carcinomas (TCs) ${ }^{8,9)}$ The differential diagnosis of such tumors is important since tumors with a variety of histological types can occur in the anterior mediastinum. ${ }^{10)}$ We investigated the usefulness of positron-emission tomography (PET)/ computed tomography (CT) in the differential diagnosis and management of anterior mediastinum tumors.

\section{Methods}

The present study included 181 patients who were histologically diagnosed with an anterior mediastinal tumor between May 2006 and December 2015. One hundred 
five of these patients received PET/CT. Masaoka's stage classification $^{11)}$ and the World Health Organization (WHO) histological classification ${ }^{12}$ ) were used to classify resected thymomas. We divided the five WHO histological subtypes of thymoma into low-grade (Types A, $\mathrm{AB}$, and B1) and high-grade (Types B2 and B3) subgroups according to previous reports. ${ }^{13,14)}$

Diagnostic and therapeutic strategies for anterior mediastinal tumors at our hospital were as follows: 1) Primarily radiological follow-up or sometimes surgical resection to confirm the pathological diagnosis was selected in cystic lesions. 2) Surgical resection without performing a preoperative biopsy was selected cases suspected of having non-invasive thymic epithelial tumors. 3) CT guided biopsy was selected for massive invasive tumors and non-invasive tumors which needed to be ruled out as being either malignant lymphoma or germ cell tumors. 4) Thymic limited resection (thymomectomy or hemi-thymectomy) was considered for cases suspected of being non-invasive and low-grade type thymoma (Types A, AB, and B1).

None of the patients had diabetes, and the serum glucose levels of all patients just before PET/CT examination were less than $130 \mathrm{mg} / \mathrm{dl}$. All of the FDG-PET examinations were performed using a Biograph16 (Siemens/CTI Inc., Knoxville, TN, USA) system. All of the patients fasted for 5 hours before the tracer injection (185 MBq) for FDG-PET. The FDG-PET images were obtained at 60 minutes (early phase) after the injection. A region of interest (ROI) was drawn over each of the main masses that were evident on trans-axial images, and the maximum standardized uptake values (SUV max) were calculated.

Statistical analyses were performed using the SPSS software program (SPSS Inc, Chicago, IL, USA). All of the data were expressed as mean \pm standard deviation (SD). The differences between groups were analyzed using a two-tailed Student's $t$-test. $P$-values of $\leq 0.05$ were considered to indicate statistically significant.

\section{Results}

The mean age of the patients was $56.0 \pm 4.2$ years (range, 21-79 years). Fifty-five patients were male, and 50 were female. Sixty-eight patients underwent surgery without a preoperative biopsy, and 22 patients underwent surgery after a biopsy. The histological findings were as follows: thymoma $(n=49)$, thymic carcinoma (TC) $(\mathrm{n}=19)$, malignant lymphoma (ML) $(\mathrm{n}=8)$,

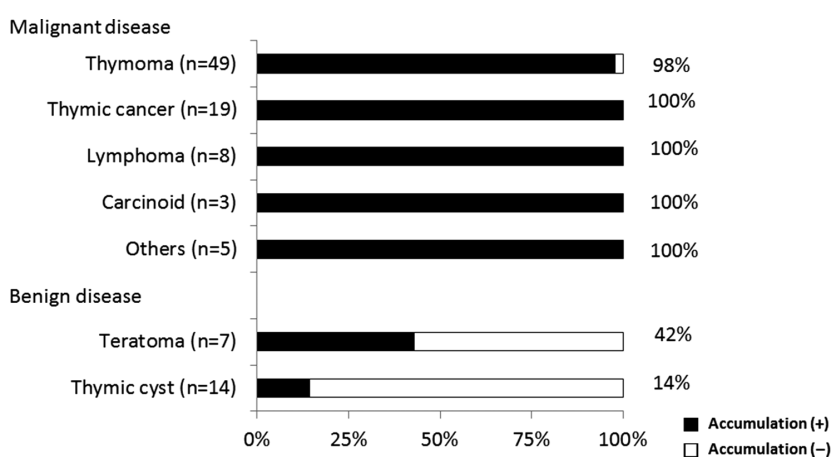

Fig. 1 FDG accumulation in an anterior mediastinal tumor. Upon visual inspection (SUV max: >2.0), all malignant tumor showed ${ }^{18} \mathrm{~F}$-FDG accumulation (with the exception of one Type A thymoma). Two of 14 (14\%) thymic cysts and three of seven (42\%) teratomas showed slight ${ }^{18}$ F-FDG accumulation. FDG: fluorodeoxyglucose; SUV max: maximum standardized uptake values; ${ }^{18} \mathrm{~F}-\mathrm{FDG}$ : ${ }^{18} \mathrm{~F}$-fluorodeoxyglucose

carcinoid tumor $(n=3)$, germ cell tumor $(\mathrm{GCT})(\mathrm{n}=2)$, liposarcoma $(n=2)$, adenocarcinoma (colonic type) $(n=1)$, teratoma $(n=7$; mature, $n=5$; immature, $n=2)$, and thymic cyst $(n=14)$. Six patients with thymoma were associated with myasthenia gravis. The WHO classifications of the thymomas were as follows: Type A $(n=1)$, Type AB $(n=14)$, Type B1 $(n=13)$, Type B2 $(n=14)$, and Type B3 $(n=7)$. The Masaoka's stage classifications of the thymomas were as follows: Stage I $(n=24)$, Stage II $(n=19)$, Stage III $(n=4)$, and Stage IV $(n=2)$. The maximum thymoma size ranged from 21 to $160 \mathrm{~mm}$ (mean, $59.2 \pm 22.6 \mathrm{~mm}$ ), Table 1 .

The histological diagnoses of the TCs were as follows: squamous cell carcinoma (SCC) $(\mathrm{n}=15)$, large cell neuroendocrine cell carcinoma (LCNEC) $(n=2)$, and poorly differentiated carcinoma $(n=2)$. Seven of the 19 patients with TC were found to have distant metastases at the initial diagnosis.

Upon visual inspection (SUV max: $>2.0$ ), all of the malignant tumors that were categorized as thymomas showed ${ }^{18} \mathrm{~F}-\mathrm{FDG}$ accumulation (with the exception of one Type A thymoma). Two of 14 thymic cysts and three of 7 teratomas showed slight ${ }^{18} \mathrm{~F}-\mathrm{FDG}$ accumulation (Fig. 1).

The SUV max values of the low-grade thymomas, high-grade thymomas, TCs, and MLs were $3.14 \pm 0.73$, $4.34 \pm 1.49,8.59 \pm 3.05$, and $10.08 \pm 2.53$, respectively with significant differences between the low-grade and high-grade thymomas, and between the TCs and MLs. One case of TCs which SUV max revealed below 4.0 (2.7) was Stage I SCC combined with thymic cyst (Fig. 2). 


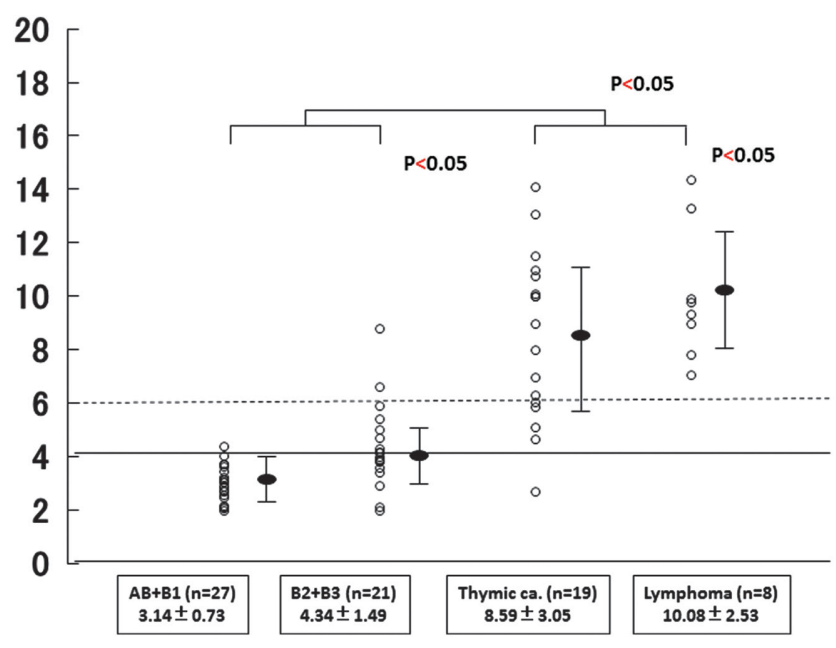

Fig. 2 The distribution of the SUV max values in anterior mediastinal tumors. The SUV max values of low-grade thymomas, high-grade thymomas, TCs and MLs were $3.14 \pm$ $0.73,4.34 \pm 1.49,8.59 \pm 3.05$ and $10.08 \pm 2.53$, respectively. Significant differences were observed between lowgrade and high-grade thymomas, and between TCs and MLs. The bars indicate mean \pm SD. MLs: malignant lymphomas; SD: standard deviation; SUV max: maximum standardized uptake values; TCs: thymic carcinomas

The SUV max of Stages I, II, and III/IV tumors were $3.12 \pm 0.92,3.53 \pm 0.89$ and $4.47 \pm 0.74$, respectively, with significant differences between Stage I and Stages II/III/IV. Receiver operating characteristic (ROC) curve of the SUV max for differentiation between low-grade and high-grade thymoma showed the cut-off value to be 3.4 (Fig. 3). Figure 4 illustrates the relationship between the SUV max and the maximum tumor diameter in patients with thymoma. The sensitivity, specificity, and accuracy of ${ }^{18} \mathrm{~F}-\mathrm{FDG}$ in the detection of low-grade thymomas and thymomas with a maximum diameter of $\leq 50 \mathrm{~mm}$ and an SUV max of $\leq 3.4$ were $85 \%, 48 \%$, and $60 \%$, respectively.

\section{Discussion}

A majority of anterior mediastinal tumors are types other than ML and apparently benign cystic lesions seem to be indicated for surgical resection. ${ }^{10,15)}$ Although thymic cysts are sometimes accompanied by tiny or histological thymomas, ${ }^{16)}$ surgery is considered to be unnecessary for many cystic lesions at the detection of the disease, and they are instead selected for radiological follow-up. However, it is sometimes difficult to differentiate thymic cysts from thymomas based on CT findings

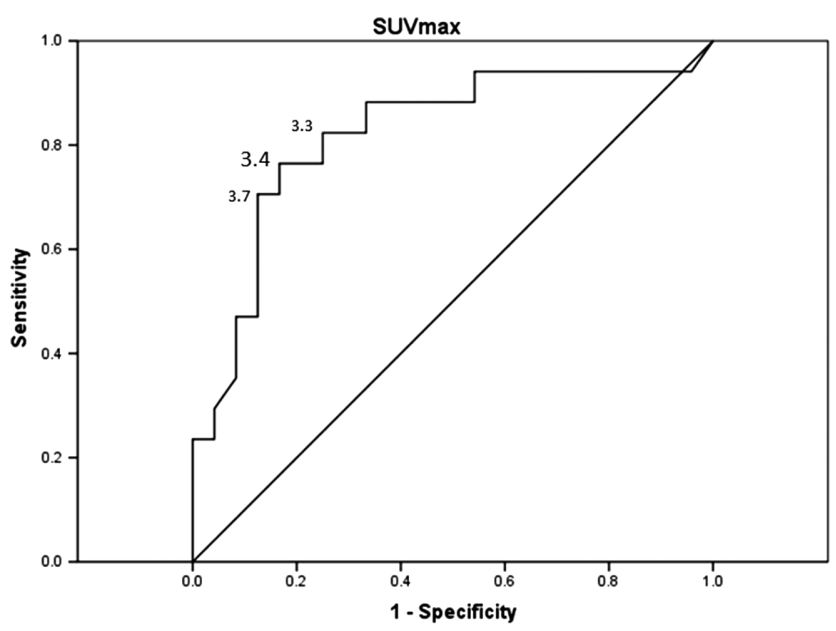

Fig. 3 ROC of the SUV max for differentiation between lowgrade and high-grade thymoma. ROC of the SUV max for differentiation between low-grade and high-grade thymoma showed the cut-off value to be 3.4. ROC: receiver operating characteristic curve; SUV max: maximum standardized uptake values

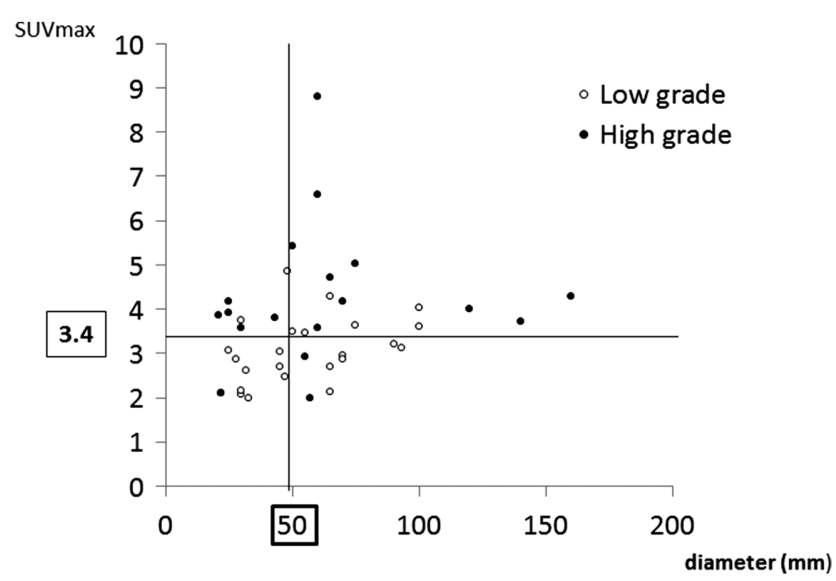

Fig. 4 The relationship between the SUV max value and the maximum thymoma diameter. The sensitivity, specificity, and accuracy of ${ }^{18} \mathrm{~F}-\mathrm{FDG}$ in the detection of low-grade thymomas and thymomas with a maximum diameter of $\leq 50 \mathrm{~mm}$ and an SUV max of $\leq 3.4$ were $85 \%, 48 \%$, and $60 \%$, respectively. SUV max: maximum standardized uptake values; ${ }^{18} \mathrm{~F}$-FDG: ${ }^{18} \mathrm{~F}$-fluorodeoxyglucose

because intracystic materials sometimes have a high viscosity. Magnetic resonance imaging (MRI) has a better contrast resolution and is more reliable than CT in detecting cystic tumors; however, some overlap between benign and malignant tumors has been demonstrated regarding the T1 and T2 parameters on MRI. ${ }^{17)}$ FDG accumulation was recognized in 48 of the 49 thymomas and in 2 of the 14 thymic cysts in this study. Thus, FDG-PET is useful for differentiating thymic cysts from thymomas. 
Table 1 Clinicopathological characteristics of patients with thymoma

\begin{tabular}{lc}
\hline Characteristics & Thymoma $(\mathrm{n}=49)$ \\
\hline Age (years) (mean \pm SD) & $56.3 \pm 18.8$ \\
Gender & 21 \\
Male & 28 \\
Female & \\
Myasthenia gravis & 6 \\
$(+)$ & 43 \\
$(-)$ & \\
Masaoka's stage & 24 \\
I & 19 \\
II & 4 \\
III & 2 \\
IV & \\
WHO classification & 1 \\
A & 14 \\
AB & 13 \\
B1 & 14 \\
B2 & 7 \\
B3 & \\
Surgical procedure & 11 \\
Extended thymectomy & 32 \\
Thymectomy & 6 \\
Hemithymectomy or partial & \\
resection of thymus & \\
\hline
\end{tabular}

WHO: World Health Organization

The definitive pathological diagnosis of ML in the mediastinum is sometimes difficult to confirm. Furthermore, even the differentiation of ML from lymphocytic predominant thymoma using the small specimens obtained by CT-guided needle biopsy can be difficult. Nakajima et al. ${ }^{18)}$ reported that the maturation stage of the lymphoid cells in the tumor that is observed in flow cytometry is useful for the differential diagnosis of thymoma from other malignant tumors. As shown in Fig. 2, the SUV max was $\leq 6.0$ in all thymomas; however, the SUV max was $>6.0$ in all MLs. Thus, the SUV max is very useful for the differential diagnosis of thymoma from ML.

In 1999, the WHO proposed a new histological classification for thymic epithelial tumors. ${ }^{12)}$ According to this classification, thymomas were classified as Types A, AB, B1, B2, or B3. Several authors have reported that the WHO classification is an independent, prognostic factor when Type A-B1 thymomas are grouped as low risk and Type B2-C thymomas are grouped as high risk. ${ }^{13,14)}$ In the clinical setting, the WHO histological classification is useful in the preoperative assessments of patients with thymic epithelial tumors and for selecting an appropriate treatment strategy. The CT and MRI findings of thymic epithelial tumors help in differentiating the subtypes of the WHO histological classification; however, there are many overlapping features.

The mean SUV max values of low-grade thymomas, high-grade thymomas, and TCs have been reported to vary ranging from 2.0 to $4.0,4.0$ to 7.4 , and 7.6 to 10.5 , respectively. ${ }^{1-3,7)}$ In this study, the SUV max values of low-grade thymomas, high-grade thymomas and TCs were $3.14 \pm 0.73,4.34 \pm 1.49$, and $8.59 \pm 3.05$, respectively; with significant differences between the low-grade and high-grade thymomas, and between the TCs. These results revealed that the SUV max is useful for differentiating the grade of malignancy in thymic epithelial tumors.

The indications for the preoperative biopsy of anterior mediastinal tumors are controversial. As shown in Fig. 2, the SUV max values were significantly higher in MLs than in TCs; however, many cases overlapped.

Anterior mediastinal tumors with an SUV max of $>6.0$ are likely to be TCs or MLs, those tumors should be biopsied to determine the therapeutic strategy. Meanwhile, as all anterior mediastinal tumors with SUV max values of 2.0-4.0 excluded extraordinary case of thymic carcinoma were thymomas, a preoperative biopsy is unnecessary for these tumors.

Surgical resection is considered to be the principal treatment for patients with thymoma; however, the optimal mode of resection in patients without myasthenia gravis (MG) is unclear. Recent guidelines have recommended complete surgical resection of the entire thymus gland (thymothymectomy). ${ }^{19)}$ On the other hand, several recent studies note that thymothymectomy might not always be necessary in non-myasthenic thymoma patients, because there were no differences in the surgical results of patients who underwent thymomectomy with or without extended thymectomy. ${ }^{20-22)}$ Thus, the optimal mode of thymoma resection in non-myasthenic thymoma patients remains controversial, and the oncological indication of limited resection (thymomectomy) is unclear.

As mentioned before, several authors have reported that the prognosis of low-grade thymomas is better than that of high grade thymomas. Weis et al. ${ }^{23)}$ reported that WHO histology is an independent prognostic factor in recurrence but not survival based on the International Thymic Malignancy Interest Group (ITMIG) retrospective database.

We assumed that FDG-PET/CT may be useful in aiding the decisions regarding the limited resection of thymomas. We found that $85 \%$ of thymomas with a maximum diameter of $\leq 50 \mathrm{~mm}$ and an SUV max of $\leq 3.4$ were low-grade thymomas. This finding may be an 
oncological criterion for limited resection. The present study is associated with some limitations, including its retrospective design and the small number of surgical cases.

\section{Conclusion}

As anterior mediastinal tumors with an SUV max of $>6.0$ are likely to be TCs or MLs, those tumors should be biopsied to determine the therapeutic strategy. As many anterior mediastinal solid tumors with a maximum diameter of $\leq 50 \mathrm{~mm}$ and an SUV max of $\leq 3.4$ were low-grade thymomas, this finding may be an oncological criterion for limited resection. FDG-PET/CT is an objective and useful modality in the differential diagnosis and management of anterior mediastinal tumors.

\section{Disclosure Statement}

There are no companies, etc. in a relation of conflict of interest requiring disclosure in relation to this manuscript.

\section{References}

1) Sung YM, Lee KS, Kim BT, et al. 18-F FDG PET/CT of thymic epithelial tumors: usefulness for distinguishing and staging tumor subgroups. J Nucl Med 2006; 47: 1628-34.

2) Endo M, Nakagawa K, Ohde $Y$, et al. Utility of 18FDG-PET for differentiating the grade of malignancy in thymic epithelial tumors. Lung Cancer 2008; 61: $350-5$.

3) Inoue A, Tomiyama N, Tatsumi M, et al. (18)F-FDG PET for the evaluation of thymic epithelial tumors: Correlation with the World Health Organization classification in addition to dual-time-point imaging. Eur J Nucl Med Mol Imaging 2009; 36: 1219-25.

4) Terzi A, Bertolaccini L, Rizzardi G, et al. Usefulness of 18-F FDG PET/CT in the pre-treatment evaluation of thymic epithelial neoplasms. Lung Cancer 2011; 74: 239-43.

5) Terzi A, Bertolaccini L, Rizzardi G, et al. Usefulness of 18-F FDG PET/CT in the pre-treatment evaluation of thymic epithelial neoplasms. Lung Cancer 2011; 74: 239-43.

6) Nakajo M, Kajiya Y, Tani A, et al. ${ }^{18}$ FDG PET for grading malignancy in thymic epithelial tumors: significant differences in ${ }^{18} \mathrm{FDG}$ uptake and expression of glucose transporter-1 and hexokinase II between low and high-risk tumors: preliminary study. Eur J Radiol 2012; 81: 146-51.

7) Otsuka H. The utility of FDG-PET in the diagnosis of thymic epithelial tumors. J Med Invest 2012; 59: 225-34.
8) Kubota K, Yamada S, Kondo T, et al. PET imaging of primary mediastinal tumours. Br J Cancer 1996; 73: 882-6.

9) Kaira K, Abe M, Nakagawa K, et al. ${ }^{18}$ F-FDG uptake on PET in primary mediastinal non-thymic neoplasm: a clinicopathological study. Eur J Radiol 2012; 81: 2423-9.

10) Kitami A, Suzuki T, Usuda R, et al. Diagnostic and therapeutic thoracoscopy for mediastinal disease. Ann Thorac Cardiovasc Surg 2004; 10: 14-8.

11) Masaoka A, Monden Y, Nakahara K, et al. Follow-up study of thymomas with special reference to their clinical stages. Cancer 1981; 48: 2485-92.

12) Travis WD, Brambillia E, Mueller-Hermelink HK, Harris CC. WHO classification of tumors. Pathology and genetics of tumors of the lung, pleura, thymus and heart. Lyon: IARC Press; 2004.

13) Okumura M, Ohta M, Tateyama H, et al. The World Health Organization histologic classification system reflects the oncologic behavior of thymoma: a clinical study of 273 patients. Cancer 2002; 94: 624-32.

14) Chen G, Marx A, Chen WH, et al. New WHO histologic classification predicts prognosis of thymic epithelial tumors: a clinicopathologic study of 200 thymoma cases from China. Cancer 2002; 95: 420-9.

15) Kitami A, Suzuki T, Suzuki S, et al. Tiny thymic carcinoma completely surrounded by thymic tissue. The possibility of de novo carcinoma. Jpn J Thorac Cardiovasc Surg 2000; 48: 670-2.

16) Kitami A, Kamio Y, Uematsu S, et al. Thymoma with intracystic dissemination arising in a unilocular thymic cyst. Gen Thorac Cardiovasc Surg 2007; 55: 281-3.

17) Link KM, Samuels LJ, Reed JC, et al. Magnetic resonance imaging of the mediastinum. J Thorac Imaging 1993; 8: 34-53.

18) Nakajima J, Takamoto S, Oka T, et al. Flow cytometric analysis of lymphoid cells in thymic epithelial neoplasms. Eur J Cardiothorac Surg 2000; 18: 287-92.

19) National Comprehensive Cancer Network. NCCN Clinical Practice Guidelines in Oncology - Thymomas and thymic carcinomas. V.2.2013. Avaliable at: http://www.nccn.org/professionals/physician_gls/ PDF/thymic.pdf.

20) Falkson CB, Bezjak A, Darling G, et al. The management of thymoma: a systematic review and practice guideline. J Thorac Oncol 2009; 4: 911-9.

21) Tseng YC, Hsieh CC, Huang HY, et al. Is thymectomy necessary in nonmyasthenic patients with early thymoma? J Thorac Oncol 2013; 8: 952-8.

22) Nakagawa K, Asamura H, Sakurai H, et al. Does the mode of surgical resection affect the prognosis/recurrence in patients with thymoma? J Surg Oncol 2014; 109: $179-83$.

23) Weis CA, Yao X, Deng Y, et al. The impact of thymoma histotype on prognosis in a worldwide database. J Thorac Oncol 2015; 10: 367-72. 